\title{
Different Stellar Rotations in the Two Main Sequences of the Young Globular Cluster NGC 1818: The First Direct Spectroscopic Evidence*
}

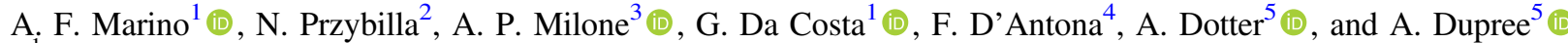 \\ ${ }^{1}$ Research School of Astronomy \& Astrophysics, Australian National University, Canberra, ACT 2611, Australia; anna.marino@anu.edu.au \\ 2 Institut für Astro- und Teilchenphysik, Universität Innsbruck, Technikerstrasse 25, A-6020, Innsbruck, Austria \\ ${ }^{3}$ Dipartimento di Fisica e Astronomia “Galileo Galilei”-Univ. di Padova, Vicolo dell'Osservatorio 3, Padova, I-35122, Italy \\ ${ }^{4}$ INAF-Osservatorio Astronomico di Roma, Monte Porzio, Italy \\ ${ }^{5}$ Harvard-Smithsonian Center for Astrophysics, 60 Garden Street, Cambridge, MA 02138, USA \\ Received 2018 May 21; revised 2018 July 11; accepted 2018 July 11; published 2018 August 23
}

\begin{abstract}
We present a spectroscopic analysis of main sequence (MS) stars in the young globular cluster NGC 1818 (age $\sim 40 \mathrm{Myr}$ ) in the Large Magellanic Cloud. Our photometric survey of Magellanic Clouds clusters has revealed that NGC 1818, similar to other young objects with ages $\lesssim 600 \mathrm{Myr}$, displays not only an extended MS turnoff (eMSTO), as observed in intermediate-age clusters (age $\sim 1-2$ Gyr), but also a split MS. The most straightforward interpretation of the double MS is the presence of two stellar populations: a sequence of slowly rotating stars lying on the blue-MS (bMS) and a sequence of fast rotators, with rotation close to the breaking speed, defining a red-MS (rMS). We report the first direct spectroscopic measurements of projected rotational velocities $v$ sin $i$ for the double MS, eMSTO, and Be stars of a young cluster. The analysis of line profiles includes non-local thermodynamic equilibrium effects, required for correctly deriving $v \sin i$ values. Our results suggest that: (i) the mean rotation for bMS and rMS stars is $v \sin i=71 \pm 10 \mathrm{~km} \mathrm{~s}^{-1}\left(\sigma=37 \mathrm{~km} \mathrm{~s}^{-1}\right)$ and $v \sin i=202 \pm 23 \mathrm{~km} \mathrm{~s}^{-1}\left(\sigma=91 \mathrm{~km} \mathrm{~s}^{-1}\right)$, respectively; (ii) eMSTO stars have different $v \sin i$, which are generally lower than those inferred for rMS stars, and (iii) as expected, Be stars display the highest $v \sin i$ values. This analysis supports the idea that distinct rotational velocities play an important role in the appearance of multiple stellar populations in the color-magnitude diagrams of young clusters, and poses new constraints on the current scenarios.
\end{abstract}

Key words: Hertzsprung-Russell and C-M diagrams - Magellanic Clouds - stars: rotation - star clusters: individual (NGC 1818)

\section{Introduction}

In the last decade, several papers based on high-precision photometry have revealed that the color-magnitude diagram (CMD) of most old Galactic globular clusters (GGCs) is made of multiple sequences (e.g., Milone et al. 2017b). These sequences have been observed along the entire CMD and correspond to distinct stellar populations with different $\mathrm{He}$ and $\mathrm{C}, \mathrm{N}, \mathrm{O}, \mathrm{Na}$ abundance (e.g., Marino et al. 2008, 2016; Milone et al. 2015b). To explain the observations, some authors have suggested that GCs have experienced a prolonged star formation history (e.g., Decressin et al. 2007; D'Antona et al. 2016), while other works have concluded that stars in GCs are coeval and that the observed multiple populations are due to disk accretion in premain sequence (MS) stars (e.g., Bastian et al. 2013). In general, the series of events that led from massive gas clouds in the early universe to the GCs we see today, with their multiple populations, remains a puzzle (Renzini et al. 2015).

Multiple stellar populations are not a peculiarity of old Milky Way GCs. In the last decade high-accuracy photometry from the Hubble Space Telescope (HST) has revealed that several 1-2 Gyr old star clusters in both Magellanic Clouds (MCs) exhibit bimodal or extended MS turnoffs (eMSTOs) and dual clumps (Mackey \& Broby Nielsen 2007; Glatt et al. 2008; Girardi et al. 2009; Milone et al. 2009). In particular, our HST survey of star clusters in the MCs has shown that the eMSTO is a common feature of

\footnotetext{
* Based on observations collected at the European Organisation for Astronomical Research in the Southern Hemisphere under ESO programme 0100.D-0520(A), and observations with the NASA/ESA Hubble Space Telescope, obtained at the Space Telescope Science Institute, which is operated by AURA, Inc., under NASA contract NAS 5-26555.
}

intermediate-age star clusters, as it has been observed in the majority of the analyzed clusters (Milone et al. 2009). Several authors have thus suggested that eMSTO clusters are the counterparts of the old GCs with multiple sequences (Conroy \& Spergel 2011; Keller et al. 2011) and that they provide a laboratory to study multiple populations of few hundreds-Myr after their formation. However, while the MSTO of most intermediate-age star clusters is widely broadened in color and magnitude, the MS seems consistent with a single isochrone (Milone et al. 2009).

More recently, double MSs have been detected in very young clusters, with ages between $\sim 50$ and $\sim 600 \mathrm{Myr}$ (Milone et al. 2015a, 2016, 2017a, 2018; Correnti et al. 2017). These young GCs not only exhibit an eMSTO, but differing from the intermediateage clusters, they also host a split MS, similar to that observed in Milky Way globulars (e.g., Milone et al. 2012; Milone 2015).

The most straightforward interpretation of the eMSTO was an extended star formation history with a duration of 100-500 Myr (e.g., Milone et al. 2009; Goudfrooij et al. 2014). According to this scenario, young and intermediate-age GCs have experienced a complex star formation history, with two or more stellar generations. This in turn would suggest that young and intermediate-age MC clusters could be the counterparts of old GGCs with multiple stellar populations. As an alternative, Bastian \& de Mink (2009) suggested that stellar rotation can mimic an age spread and an eMSTO.

Comparison of the CMDs of young clusters with isochrones suggests that the split MS is best reproduced by two stellar populations with distinct rotation: a non-rotating population of blue MS (bMS) stars and a population of red MS (rMS) stars with rotational velocities close to the critical value 
(D'Antona et al. 2015; Milone et al. 2016). One of the most crucial topics in the field of multiple stellar populations is to understand if the double MSs in young GCs are the counterparts of those observed on old Milky Way globulars or are instead a different phenomenon.

Direct detection of rapidly rotating stars among the eMSTOs was provided by Dupree et al. (2017) from an analysis of highresolution spectra of eMSTO stars in the young cluster NGC 1866. In this work we analyze spectroscopic data for stars in the GC NGC 1818 in the Large MC (LMC), in order to estimate the projected rotational velocity $v \sin i$. NGC $1818^{6}$ $\left(\log \left(M / M_{\odot}\right)=4.41\right)$, with an age of $\sim 40 \mathrm{Myr}$, is one of the very young GCs with a detected split MS (Milone et al. 2018), interpreted as having a $\sim 30 \%$ composition of non-rotating stars on the bMS, and a $\sim 70 \%$ composition of fast rotators with rotation $\omega \sim 0.9 \omega_{\text {crit }}$ on the rMS, in the mag range $18.2 \lesssim$ $m_{\mathrm{F} 814 \mathrm{~W}} \lesssim 20.7$. An eMSTO plus a population of Be stars with mag $15.5 \lesssim m_{\mathrm{F} 814 \mathrm{~W}} \lesssim 18.5$ have been also detected. The layout of this paper is as follows. Section 2 describes the photometric and spectroscopic data; our results are presented in Section 3 and discussed in Section 4.

\section{Data}

\subsection{The Photometric Data Set}

To investigate multiple sequences along the CMD of NGC 1818, we used the photometric and astrometric catalog published by Milone et al. (2018). This catalog includes HST photometry of NGC 1818 stars in the F336W, F656N, and F814W bands of the Ultraviolet and Visual Channel of the Wide Field Camera 3. Flux and magnitude measurements have been derived from the images collected as part of GO-13727 (PI J. Kalirai) and GO-14710 (PI A. Milone) using the methods of data analysis and data reduction developed by Jay Anderson. Photometry has been calibrated to the VEGA-mag system using the photometric zero-points provided on the WFC3/ UVIS web page (see Bedin et al. 2005 for details), while stellar positions are transformed in the Gaia data release 1 reference frame (Gaia Collaboration et al. 2016). We refer the interested reader to the works by Milone et al. (2018), Anderson et al. (2008) and references therein for details on the database and on the data reduction.

\subsection{The Spectroscopic Data Set}

The spectroscopic data consist of FLAMES/GIRAFFE data (Pasquini et al. 2002) collected under the ESO programme 0100.D-0520 (PI: Marino) taken in Visitor mode during the nights 2017 December 13-15.

The GIRAFFE fibers were used with the HR15N setup, covering the spectral range from $\sim 647$ to $\sim 679 \mathrm{~nm}$ with a resolving power of $R=\lambda / \Delta \lambda \sim 19,200$. We have observed a total of 44 stars in the $2 ! 7 \times 2 ! 7$ field of view around NGC 1818 using two FLAMES configurations. The two configurations were observed in 15 and 13 different exposures of $\sim 46$ minutes. Data reduction involving bias subtraction, flatfield correction, wavelength calibration, sky subtraction, and continuum normalization has been done using the dedicated pipelines for GIRAFFE and IRAF ${ }^{7}$ standard procedures. At the

\footnotetext{
6 Taken from McLaughlin \& van der Marel (2005) by assuming a Wilson (1975) profile.

7 IRAF is distributed by NOAO, which is operated by AURA, under cooperative agreement with the US National Science Foundation.
}

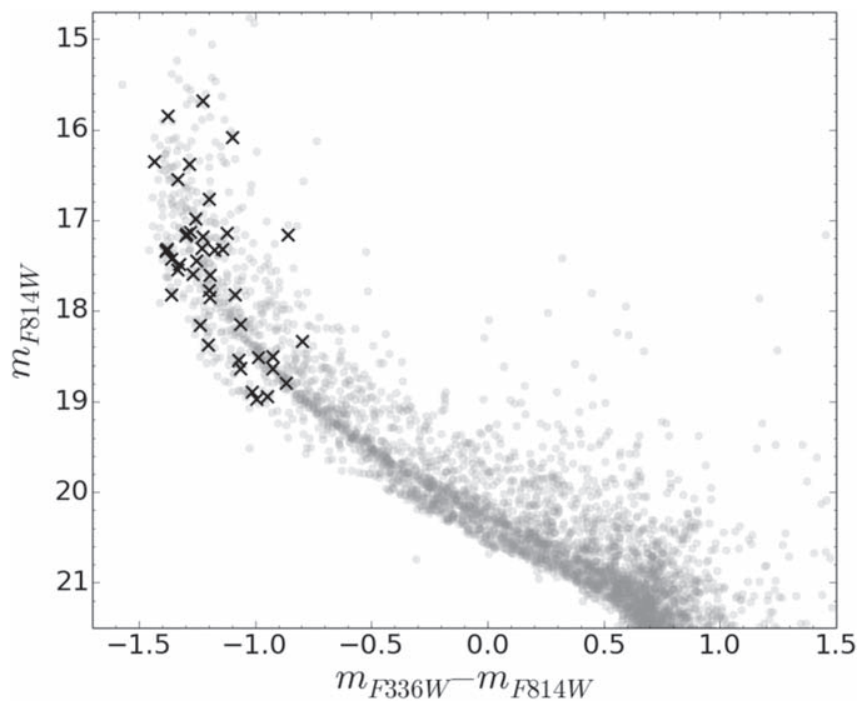

Figure 1. $m_{\mathrm{F} 814 \mathrm{~W}}-\left(m_{\mathrm{F} 336 \mathrm{~W}}-m_{\mathrm{F} 814 \mathrm{~W}}\right) \mathrm{CMD}$ for NGC 1818. Our spectroscopic targets are represented with black crosses.

wavelength of the He I $6678 \AA$ line, the typical signal-to-noise ratio $(\mathrm{S} / \mathrm{N})$ of the fully reduced and combined spectra ranges from $\sim 30$ for the faintest targets to $\sim 80$ for the brightest ones.

The observed stars, shown in Figure 1, have been selected from the CMD described in Section 2.1. Specifically, we have observed stars on both the bMS and rMS, plus some objects in the eMSTO region, and 4 stars classified as Be (see Section 3.3 for further details on their selection). A few stars are observed with a redder color with respect to the rMS, where binaries are expected. In the following we discuss the observed stars on the basis of their location on the CMD, plus spectroscopic information. Hence, bona fide bMS and rMS stars will be discussed separately from the eMSTO and Be stars. Stars clearly showing double-line features in their spectra are classified as binaries. It is noteworthy to clarify that, although our high-precision photometry allowed us to properly select stars on different regions of the CMD with small errors, a residual contamination is expected. In particular, from the CMD in Figure 1, it is clear that there could be some overlap between the rMS, binaries, and/or Be stars. To avoid confusion, we keep this selection throughout the paper, and discuss in each section the presence of possible contaminants.

The stellar spectral lines covered with the HR15N setup in the sample stars are $\mathrm{H} \alpha$, He I $\lambda 6678 \AA$, and in some cases, the C II doublet $\lambda \lambda 6578 / 82 \AA$. In addition, spatially variable sharp nebular emission lines are visible, comprising $\mathrm{H} \alpha,[\mathrm{N} \mathrm{II}] \lambda \lambda 6548 / 84 \AA$, and [S II] $\lambda \lambda 6717 / 31 \AA$, particularly in the fainter objects.

Heliocentric radial velocities $v_{\mathrm{r}, \odot}$ of the sample stars were determined by cross-correlation of the observed spectra with appropriate synthetic spectra (see below). Overall, the precision of the $v_{\mathrm{r}, \odot}$-determination for the individual star is influenced by the number of useful lines $(\mathrm{H} \alpha$ and/or He I $\lambda 6678 \AA$ were considered), the rotational broadening (sharper lines giving more precise results), and the presence and location of the nebular emission features. After application of a $2 \sigma$-clipping criterion with an iteration depth of 2,33 out of 39 stars with $v_{\mathrm{r}, \odot}$ data remain for the determination of the cluster radial velocity of $311.1 \pm 3.9 \mathrm{~km} \mathrm{~s}^{-1}(1 \sigma$-scatter $)$. The outliers, stars \#27, \#28, \#96, \#57, and \#77, which belong to our rMS and bMS selection, are assumed to be single-lined spectroscopic binaries (SB1) candidates. However, due to their similarly high 
Table 1

Observation Details, Coordinates, and Radial Velocities for Our Spectroscopic Targets

\begin{tabular}{|c|c|c|c|c|c|c|c|c|}
\hline ID & $\alpha(2000)$ & $\delta(2000)$ & $m_{\mathrm{F} 336 \mathrm{~W}}$ & $m_{\mathrm{F} 656 \mathrm{~N}}$ & $m_{\mathrm{F} 814 \mathrm{~W}}$ & $\mathrm{RV}_{\text {helio }}\left(\mathrm{km} \mathrm{s}^{-1}\right)$ & $v \sin i\left(\mathrm{~km} \mathrm{~s}^{-1}\right)$ & CMD Region \\
\hline 31 & 05:04:03.72 & $-66: 25: 30.8$ & 16.3236 & 17.2822 & 17.591 & $311 \pm 2$ & $108 \pm 2$ & redMS \\
\hline 33 & 05:04:14.16 & $-66: 25: 34.0$ & 15.8604 & 16.8372 & 17.155 & $314 \pm 3$ & $194 \pm 4$ & redMS \\
\hline 83 & 05:04:17.44 & $-66: 26: 17.0$ & 15.8471 & 16.7573 & 17.127 & $310 \pm 2$ & $155 \pm 7$ & redMS \\
\hline 117 & 05:04:18.22 & $-66: 26: 40.2$ & 16.4095 & 17.3158 & 17.603 & $307 \pm 2$ & $193 \pm 5$ & redMS \\
\hline 102 & 05:04:10.48 & $-66: 26: 24.7$ & 16.0832 & 16.9004 & 17.311 & $310 \pm 5$ & $299 \pm 7$ & redMS \\
\hline 141 & 05:04:00.86 & $-66: 26: 51.2$ & 16.6612 & 17.4815 & 17.855 & $309 \pm 5$ & $347 \pm 6$ & redMS \\
\hline 69 & 05:04:07.76 & $-66: 26: 01.0$ & 15.8713 & 16.8311 & 17.170 & $310 \pm 2$ & $258 \pm 6$ & redMS \\
\hline 85 & 05:04:01.03 & $-66: 26: 13.3$ & 17.0840 & 17.7970 & 18.148 & $314 \pm 8$ & $312 \pm 15$ & redMS \\
\hline 26 & 05:04:14.83 & $-66: 25: 24.0$ & 17.5280 & 18.2406 & 18.514 & $315 \pm 12$ & $170 \pm 20$ & redMS \\
\hline 66 & 05:04:20.57 & $-66: 26: 03.2$ & 17.7081 & 18.3423 & 18.634 & $315 \pm 8$ & $50 \pm 10$ & redMS \\
\hline 57 & 05:04:07.91 & $-66: 25: 51.8$ & 16.1990 & 17.1258 & 17.451 & $297 \pm 6$ & $230 \pm 15$ & redMS \\
\hline 15 & 05:04:08.98 & $-66: 25: 16.7$ & 17.9261 & 18.4930 & 18.794 & $313 \pm 10$ & $80 \pm 20$ & redMS \\
\hline 147 & 05:04:03.92 & $-66: 26: 58.5$ & 17.5850 & 18.1842 & 18.510 & $315 \pm 10$ & $80 \pm 10$ & redMS \\
\hline 77 & 05:04:05.48 & $-66: 26: 07.0$ & 15.9587 & 16.8638 & 17.184 & $296 \pm 5$ & $340 \pm 10$ & redMS \\
\hline 133 & 05:04:18.22 & $-66: 26: 49.9$ & 16.1560 & 17.0691 & 17.332 & $305 \pm 3$ & $186 \pm 4$ & redMS \\
\hline 157 & 05:04:10.33 & $-66: 27: 16.0$ & 16.7341 & 17.4968 & 17.823 & $314 \pm 6$ & $185 \pm 7$ & redMS \\
\hline 27 & 05:04:13.42 & $-66: 25: 20.9$ & 17.5688 & 18.3155 & 18.632 & $323 \pm 8$ & $100 \pm 15$ & blueMS \\
\hline 63 & 05:04:16.57 & $-66: 25: 47.6$ & 16.4660 & 17.4731 & 17.826 & $315 \pm 2$ & $65 \pm 5$ & blueMS \\
\hline 65 & 05:04:17.58 & $-66: 26: 00.1$ & 15.9411 & 16.9562 & 17.322 & $307 \pm 2$ & $43 \pm 2$ & blueMS \\
\hline 107 & 05:04:23.40 & $-66: 26: 30.2$ & 17.9798 & 18.7799 & 18.973 & $\ldots$ & $\ldots$ & blueMS \\
\hline 115 & 05:04:14.26 & $-66: 26: 36.3$ & 15.9530 & 17.0013 & 17.337 & $313 \pm 3$ & $143 \pm 6$ & blueMS \\
\hline 148 & 05:04:10.04 & $-66: 26: 59.9$ & 16.1658 & 17.1569 & 17.491 & $310 \pm 2$ & $45 \pm 2$ & blueMS \\
\hline 56 & 05:04:04.97 & $-66: 25: 48.8$ & 17.9942 & 18.6954 & 18.941 & $315 \pm 12$ & $115 \pm 10$ & blueMS \\
\hline 28 & 05:04:18.09 & $-66: 25: 30.5$ & 17.4755 & 18.3383 & 18.547 & $290 \pm 6$ & $85 \pm 10$ & blueMS \\
\hline 88 & 05:04:17.58 & $-66: 26: 00.1$ & 15.9411 & 16.9562 & 17.322 & $304 \pm 2$ & $29 \pm 2$ & blueMS \\
\hline 96 & 05:04:16.91 & $-66: 26: 24.6$ & 16.0693 & 17.1000 & 17.430 & $288 \pm 1$ & $34 \pm 2$ & blueMS \\
\hline 166 & 05:04:14.26 & $-66: 26: 36.3$ & 15.9530 & 17.0013 & 17.337 & $311 \pm 2$ & $115 \pm 10$ & blueMS \\
\hline 20 & 05:04:06.97 & $-66: 25: 20.5$ & 16.2154 & 17.2305 & 17.549 & $312 \pm 2$ & $62 \pm 4$ & blueMS \\
\hline 140 & 05:04:21.07 & $-66: 26: 52.1$ & 17.8749 & 18.6517 & 18.890 & $307 \pm 10$ & $20 \pm 10$ & blueMS \\
\hline 152 & 05:04:17.47 & $-66: 27: 07.2$ & 16.9257 & 17.8575 & 18.164 & $320 \pm 5$ & $55 \pm 8$ & blueMS \\
\hline 100 & 05:04:03.24 & $-66: 26: 21.5$ & 17.1726 & 18.0690 & 18.375 & $312 \pm 6$ & $76 \pm 5$ & blueMS \\
\hline 41 & 05:04:12.64 & $-66: 25: 40.2$ & 15.7297 & 16.6497 & 16.987 & $306 \pm 2$ & $245 \pm 10$ & MSTO \\
\hline 95 & 05:04:06.24 & $-66: 26: 20.6$ & 15.2109 & 16.2206 & 16.544 & $310 \pm 1$ & $9 \pm 2$ & MSTO \\
\hline 16 & 05:04:12.99 & $-66: 25: 15.7$ & 14.4708 & 15.4789 & 15.846 & $306 \pm 1$ & $70 \pm 3$ & MSTO \\
\hline 89 & 05:04:08.61 & $-66: 26: 16.2$ & 14.9200 & 16.0089 & 16.355 & $307 \pm 1$ & $31 \pm 1$ & MSTO \\
\hline 118 & 05:04:21.49 & $-66: 26: 39.9$ & 14.4558 & 15.2953 & 15.683 & $308 \pm 1$ & $105 \pm 3$ & MSTO \\
\hline 46 & 05:04:08.96 & $-66: 25: 41.2$ & 16.3038 & 16.3930 & 17.162 & $309 \pm 5$ & {$[300 \pm 10]$} & $\mathrm{Be}$ \\
\hline 156 & 05:04:15.29 & $-66: 27: 12.9$ & 16.1724 & 15.7900 & 17.315 & $318 \pm 7$ & {$[320 \pm 7]$} & $\mathrm{Be}$ \\
\hline 109 & 05:04:11.97 & $-66: 26: 34.0$ & 16.0199 & 16.6490 & 17.142 & $314 \pm 8$ & {$[380 \pm 10]$} & $\mathrm{Be}$ \\
\hline 84 & $05: 04: 20.27$ & $-66: 26: 14.8$ & 14.9870 & 14.1445 & 16.085 & $292 \pm 5$ & {$[340 \pm 14]$} & $\mathrm{Be}$ \\
\hline 131 & 05:04:11.87 & $-66: 26: 44.4$ & 15.0971 & 16.0634 & 16.381 & $\ldots$ & $\ldots$ & Binary candidate \\
\hline 40 & 05:04:07.32 & $-66: 25: 38.2$ & 15.5616 & 16.4533 & 16.762 & $\ldots$ & $\ldots$ & Binary candidate \\
\hline 130 & 05:04:07.00 & $-66: 26: 44.4$ & 17.5428 & 18.1193 & 18.339 & $\ldots$ & $\ldots$ & Binary candidate \\
\hline 36 & 05:04:19.68 & $-66: 25: 39.2$ & 16.5695 & 17.4825 & 17.769 & $\ldots$ & $\ldots$ & Binary candidate \\
\hline
\end{tabular}

$v_{\mathrm{r}, \odot}$, while considering their possible binarity, we will treat them how we treat the other rMS and bMS samples. Individual $v_{\mathrm{r}, \odot}$ corrected to the heliocentric system, together with coordinates and basic HST photometry for the observed NGC 1818 stars, are listed in Table 1.

\section{Rotation}

Projected rotational velocities $v \sin i$ of the sample stars were determined by fitting the $\mathrm{H} \alpha$ Doppler core and/or the He I $\lambda 6678 \AA$ line. For this, a grid of hybrid non-local thermodynamic equilibrium (non-LTE) model spectra was computed using the approach discussed by Nieva \& Przybilla (2007) and Przybilla et al. (2011). In brief, model atmospheres in the relevant atmospheric parameter range as deduced from the CMD were computed using the ATLAS9 code (Kurucz 1993), which assumes plane-parallel geometry, chemical homogeneity, hydrostatic, radiative equilibria, and LTE. The grid covers the effective temperature range $12,000 \leqslant T_{\text {eff }} \leqslant 23,000 \mathrm{~K}$, surface gravities $3.5 \leqslant \log g \leqslant 4.5$, and helium abundances $0.07 \leqslant y \leqslant 0.10$ (by number) at half solar metallicity, as appropriate for the young stellar population of the LMC (e.g., Korn et al. 2002, including one NGC 1818 B-type star). We account for limb-darkening by using linear limb-darkening coefficients taken from Wade \& Rucinski (1985). Non-LTE level populations were computed using DETAIL and synthetic non-LTE and LTE spectra using SURFACE (Giddings 1981; Butler \& Giddings 1985 both updated and extended by K. Butler). We employed model atoms for hydrogen and helium as described by Przybilla \& Butler (2004) and Przybilla (2005).

Both the deep and narrow $\mathrm{H} \alpha$ Doppler core, as well as the deep He I $\lambda 6678 \AA$ line, result from non-LTE effects and cannot be reproduced by LTE calculations. The upper panels of Figure 2 show a comparison of profiles for the two lines for zero rotation, in non-LTE and LTE, and also an LTE profile for 


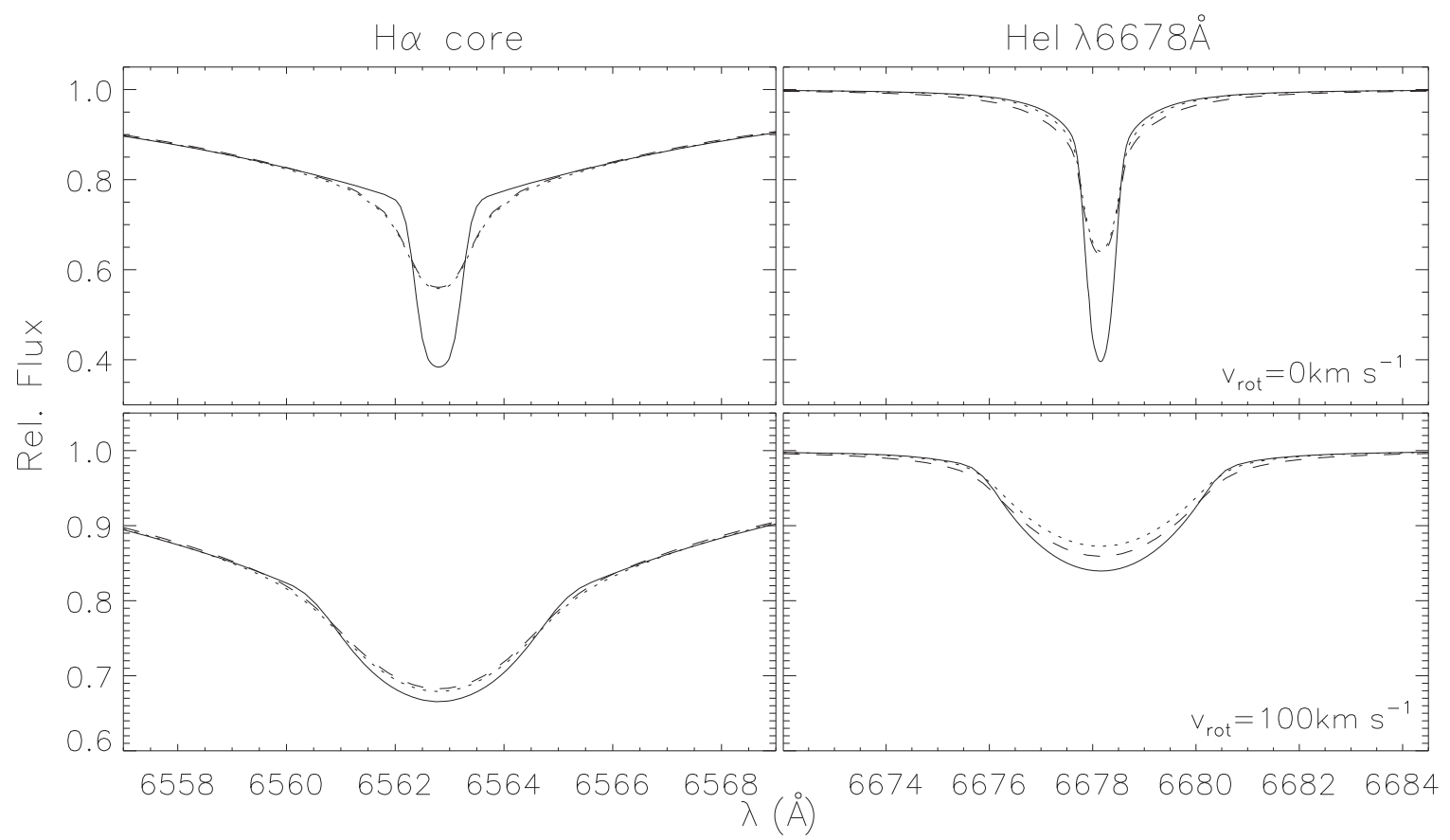

Figure 2. Upper panels: comparison of non-LTE model profiles without rotation (full lines) with LTE profiles (dotted lines) for the same atmospheric parameters ( $T_{\text {eff }}=22,000 \mathrm{~K}, \log g=4.00$ and $y=0.08$ ). A second LTE profile for $y=0.12$ (dashed line) reproduces the equivalent width of the non-LTE He I model profile. Lower panels: the same as the upper panel, but after convolution with a rotational profile for $100 \mathrm{~km} \mathrm{~s}^{-1}$.

increased helium abundance that reproduces the equivalent width of the non-LTE He I profile. The LTE line profiles are shallower and broader than the non-LTE profiles and the $\mathrm{H} \alpha$ Doppler core is largely insensitive to helium abundance changes (within reasonable limits; here 50\%). The lower panels of Figure 2 show the situation after convolution with a rotational profile for $100 \mathrm{~km} \mathrm{~s}^{-1}$. The different line profiles remain qualitatively different. An LTE analysis cannot derive consistent $v \sin i$ values from both lines simultaneously, and the values will differ from those obtained by non-LTE fits. We conclude that the consideration of non-LTE effects is essential for deriving accurate and precise $v \sin i$ values from these two lines.

We employed the Spectrum Plotting and Analysing Suite (SPAS, Hirsch 2009) for fitting model spectra to the observations. SPAS provides the means to interpolate between model grid points for up to three parameters simultaneously (here $T_{\text {eff }}$, $\log g$, and $y$ ) and allows instrumental, rotational, and (radialtangential) macrobroadening functions to be applied to the resulting theoretical profiles. The program uses the downhill simplex algorithm (Nelder \& Mead 1965) to minimize $\chi^{2}$ in order to find a good fit to the observed spectrum.

In principle, the fits provide atmospheric parameters for the sample stars. However, with only $\mathrm{H} \alpha$ and $\mathrm{He}$ I $\lambda 6678 \AA$ available as diagnostic lines, the resulting parameter values are unreliable. In the $T_{\text {eff }}$ range covered by the sample stars one does not only find normal stars but also a considerable fraction of chemically peculiar types like Bp-, HgMn-, He-weak, and Hestrong stars (e.g., Smith 1996), such that our grid covers only a fraction of the total applicable parameter space. We therefore refrain from stating atmospheric parameter values here. Fortunately, this has little impact on the $v \sin i$ determination, as the detailed parameter-dependent line shape (not the non-LTE effects, see above) gets washed out by the convolution with a rotational broadening profile already above $v \sin i$ values of $\sim 50 \mathrm{~km} \mathrm{~s}^{-1}$ for the $\mathrm{S} / \mathrm{N}$ values achieved in the observations.
Our $v \sin i$ results for the sample stars are shown in Table $1 .^{8}$ The listed uncertainties are again influenced by the same three factors as stated for the radial velocity determination in Section 2.2. The effects of reasonable atmospheric parameter uncertainties should be covered within the error bars as well. Figure 3 shows examples of the quality of the line-profile fits for the different object classes at high and low $\mathrm{S} / \mathrm{N}$ for the $v \sin i$ values given in Table 1. Note that additional uncertainties to those listed in Table 1 are introduced by the continuum settings, and are very small in the case of the slowest rotators and brightest targets, of the order of $1-2 \mathrm{~km} \mathrm{~s}^{-1}$, but can be as high as $20 \mathrm{~km} \mathrm{~s}^{-1}$ in the faintest and in the fastest rotating stars.

In addition to this, a number of effects could introduce systematic errors to the $v \sin i$ determination. Stellar variability due to, e.g., low-order (non-radial) pulsations or spots could impact the line profiles. Pulsational periods for slowly pulsating B-stars (as potentially relevant in the spectral range investigated here) and rotational periods of our sample stars are comparable to the duration of our observations (three nights). The coaddition of the individual exposures could introduce some small-scale unnoticed extra broadening of the spectral lines, mimicking higher $v \sin i$. While the effects are hard to quantify, they would imply that our $v \sin i$ values are slightly overestimated by tendency. Another effect comes from undetected binarity. This will not be relevant for the bMS stars, as their position in the CMD renders relevant second light from a companion unlikely, but the other sample stars may be affected. The additional continuum from a (fainter) companion will weaken the spectral lines of the primary, reducing their depths (but not necessarily the widths of the spectral lines of the primary). If relevant, this would result in an underestimation of $v \sin i$. Overall, the effects

\footnotetext{
8 Note that we found non-zero macroturbulent velocities for about a third of the low- $v \sin i$ stars (mostly bMS), following the procedure as discussed by Firnstein \& Przybilla (2012). The macroturbulent velocities range from about 30 to $60 \mathrm{~km} \mathrm{~s}^{-1}$, comparable to the $v \sin i$ values themselves.
} 

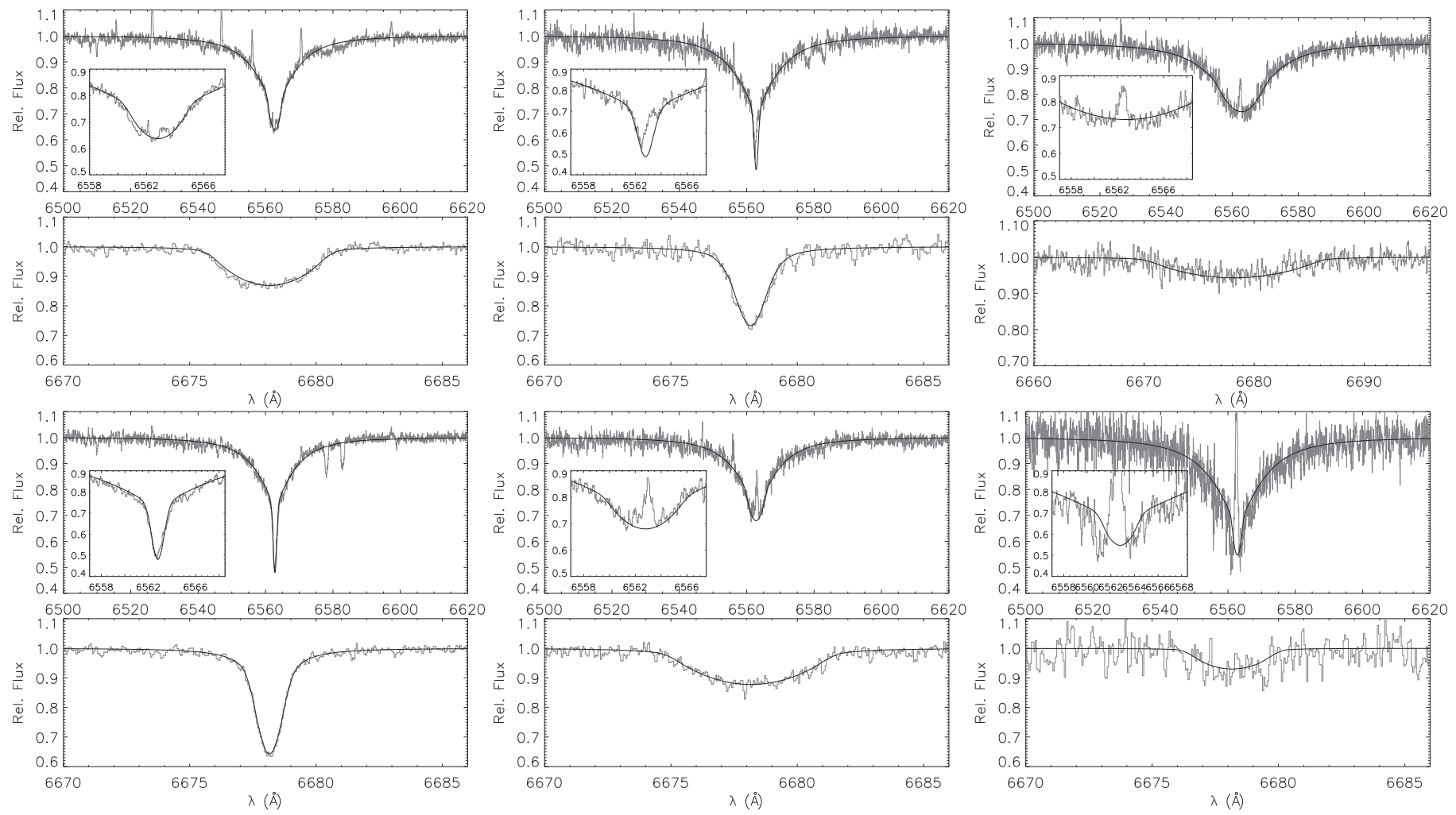

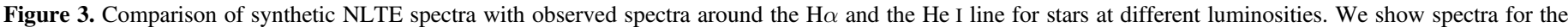

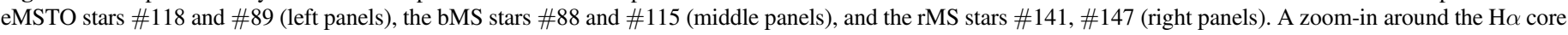
is also provided in the insets for each star.

have the potential to add some additional systematic error to the uncertainties stated in Table 1 and shift the values by some small amount, but they will not change the overall rotational velocity distribution found here, and its interpretation.

A bit of caution has to be taken in the context of the $v \sin i$ values for the Be stars, which, due to the complex profiles of their $\mathrm{H} \alpha$, were derived from the He I $\lambda 6678$ A line only. To warn the reader of the much larger uncertainties than the formal ones listed in Table $1, v \sin i$ values for Be stars have been listed in brackets. They were derived neglecting gravitationaldarkening and disk contamination, which, however, seem less important from the recent comprehensive investigation of Ahmed \& Sigut (2017) than implied previously (e.g., Townsend et al. 2004; Frémat et al. 2005). Note that the rMS stars may also be affected by gravitational-darkening to some degree, depending on how close to critical rotational velocity - in the range $\sim 400-500 \mathrm{~km} \mathrm{~s}^{-1}$ for the sample stars-they truly are.

Because of all the issues discussed above (e.g., He gravitational-settling for the slow rotators and gravitationaldarkening for faster rotators), although the He line is detectable in almost all the targets, we did not attempt any estimate of the He chemical abundance. In the following, we will separately discuss results for MS, MSTO, Be, and binaries.

\subsection{Main Sequence}

In this section we discuss the stars on the MS of NGC 1818, which include the bMS and the rMS stars. Figure 4 displays the location on the CMD of the MS stars with available spectroscopy. Our target stars are clearly distributed on the split MS of NGC 1818, and the association with the bMS and rMS is straightforward. On the side panels we show some examples of spectra for the bMS (left) and rMS (right) stars at different magnitudes, from the brightest to the faintest targets, to give an idea of the quality of our data. The spectra show both the $\mathrm{H} \alpha$ and the He I line. Note that we do not provide any $v \sin i$ for the bMS star \#107, which displays strong emission in wings. This star does not have a peculiar position on the CMD, as it is located on the bMS. The two rMS stars \#102 and \#26 display some core emission.

A qualitative inspection of this figure immediately suggests a different projected rotation for the two brightest stars, at $m_{\mathrm{F} 814 \mathrm{~W}} \sim 17.2 \mathrm{mag}$. Stars \#65 and \#69 have similar luminosity, but a different color, and as expected, the spectrum of rMS star \#69 is consistent with a significantly higher rotation than bMS \#65, as indicated by the much broadened profiles of both the $\mathrm{H} \alpha$ and the He I line. Similarly the spectral profiles of star \#85 are broader than those of the bMS star \#100, which has a similar magnitude. Lower-luminosity stars invert the trend, with rMS \#66 having lower $v \sin i$ than bMS \#27. Keeping in mind that the $v \sin i$ values are lower limits to the real rotations of the star, depending on the inclination $i$, it is more likely that a fast-rotating star has a higher $v \sin i$ than a slow rotator, but there is some possibility of the opposite being true. However, note that larger uncertainties are associated with the lower-luminosity stars with poorer $\mathrm{S} / \mathrm{Ns}$.

The mean $v \sin i$ that we obtain for the $14 \mathrm{bMSs}$ and $17 \mathrm{rMSs}$ are $\langle v \sin i\rangle_{\mathrm{bMS}}=71 \pm 10 \mathrm{~km} \mathrm{~s}^{-1}\left(\sigma=37 \mathrm{~km} \mathrm{~s}^{-1}\right)$ and $\langle v \sin i\rangle$ $\mathrm{rMS}=202 \pm 23 \mathrm{~km} \mathrm{~s}^{-1} \quad\left(\sigma=91 \mathrm{~km} \mathrm{~s}^{-1}\right)$, respectively. This means that our data are consistent with a difference in the rotation regimes of the two MSs of NGC 1818, with an average difference $\left\langle\Delta_{v \sin i_{\mathrm{rMS}}-v \sin i_{\mathrm{bMS}}}\right\rangle=+131 \pm 25 \mathrm{~km} \mathrm{~s}^{-1}$, a $>5 \sigma$ difference.

Our results for MS stars are outlined in Figure 5. The histogram distribution of $v \sin i$ for rMS and bMS stars, shown 

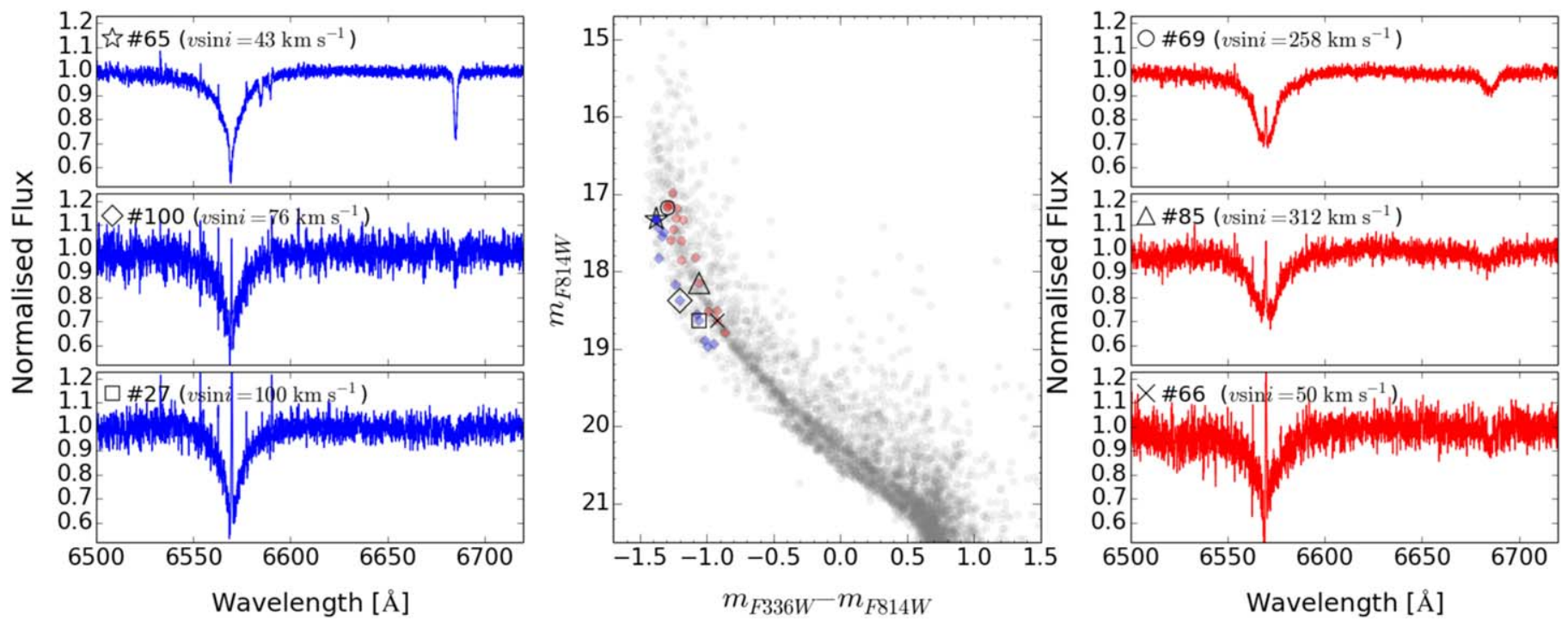

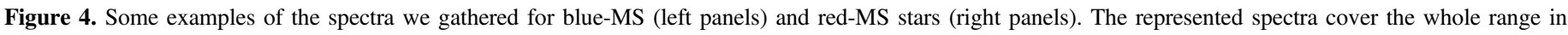

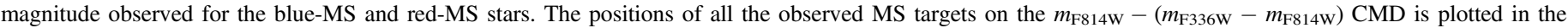
middle panel, where we explicitly indicate the location of the stars with the represented spectra on the side panels.
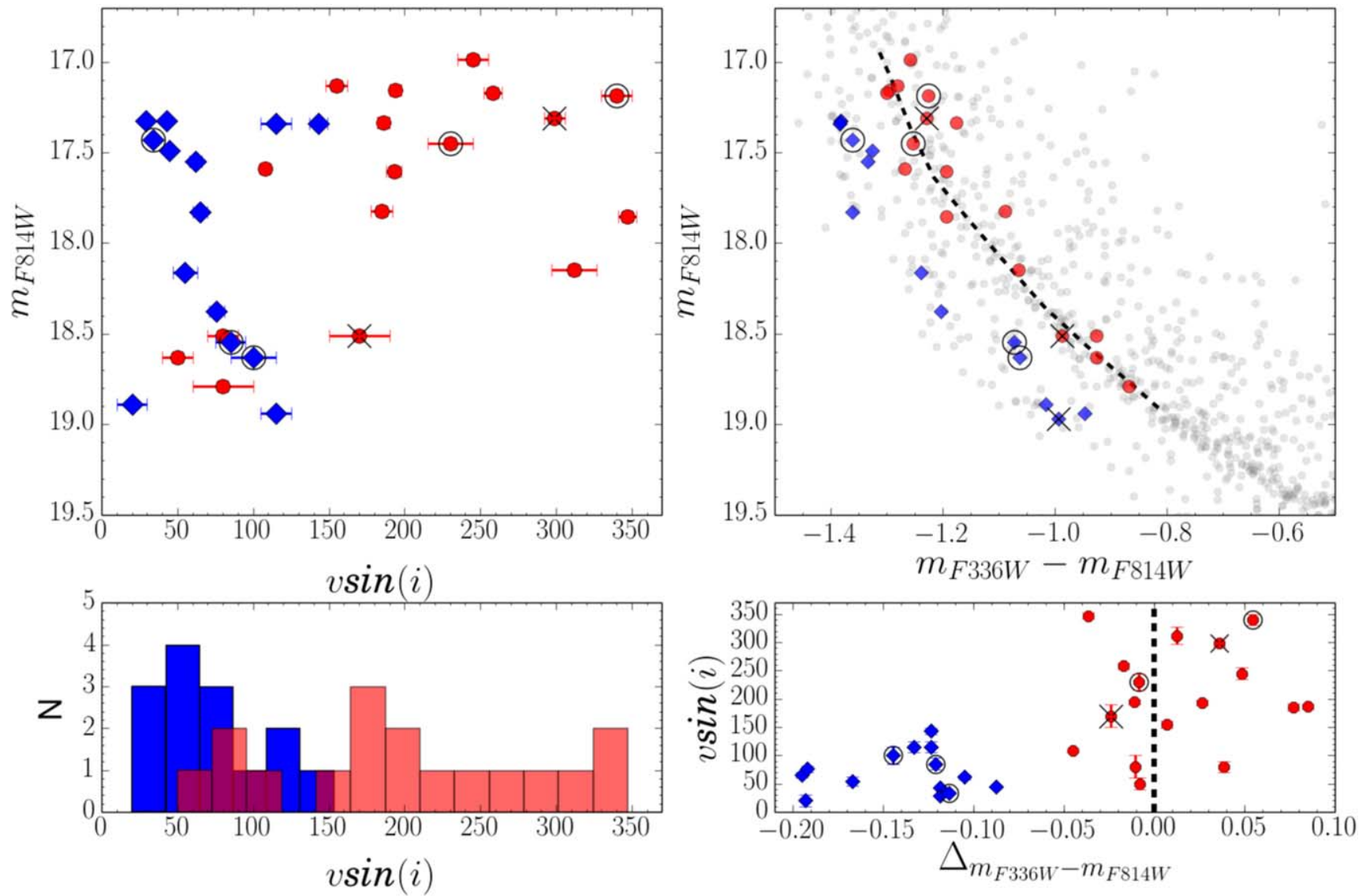

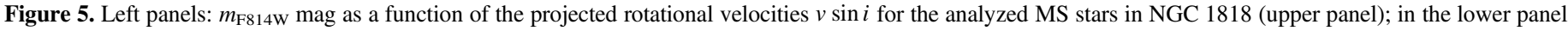

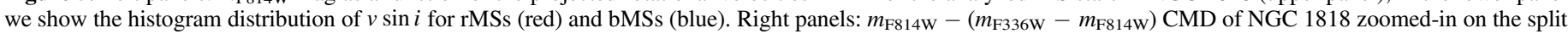

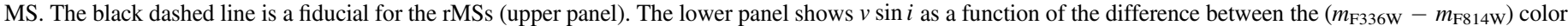

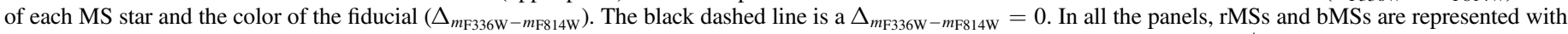

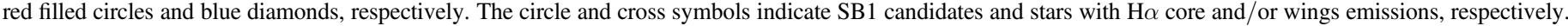

in the lower left panel, illustrates the much wider range in $v \sin i$ for the rMS stars. The upper left panel displays the $m_{\mathrm{F} 814 \mathrm{~W}}$ as a function of $v \sin i$ for rMS and bMS stars. In all the covered range in $m_{\mathrm{F} 814 \mathrm{~W}}$, bMS stars are shown to be slower rotators, but are consistent with a non-zero rotation in all cases, with some hints of higher rotation for fainter magnitudes. On the other hand, the spread in $v \sin i$ for the rMS is higher, and stars fainter than $m_{\mathrm{F} 814 \mathrm{~W}} \sim 18.5 \mathrm{mag}$ seem to rotate slower, 

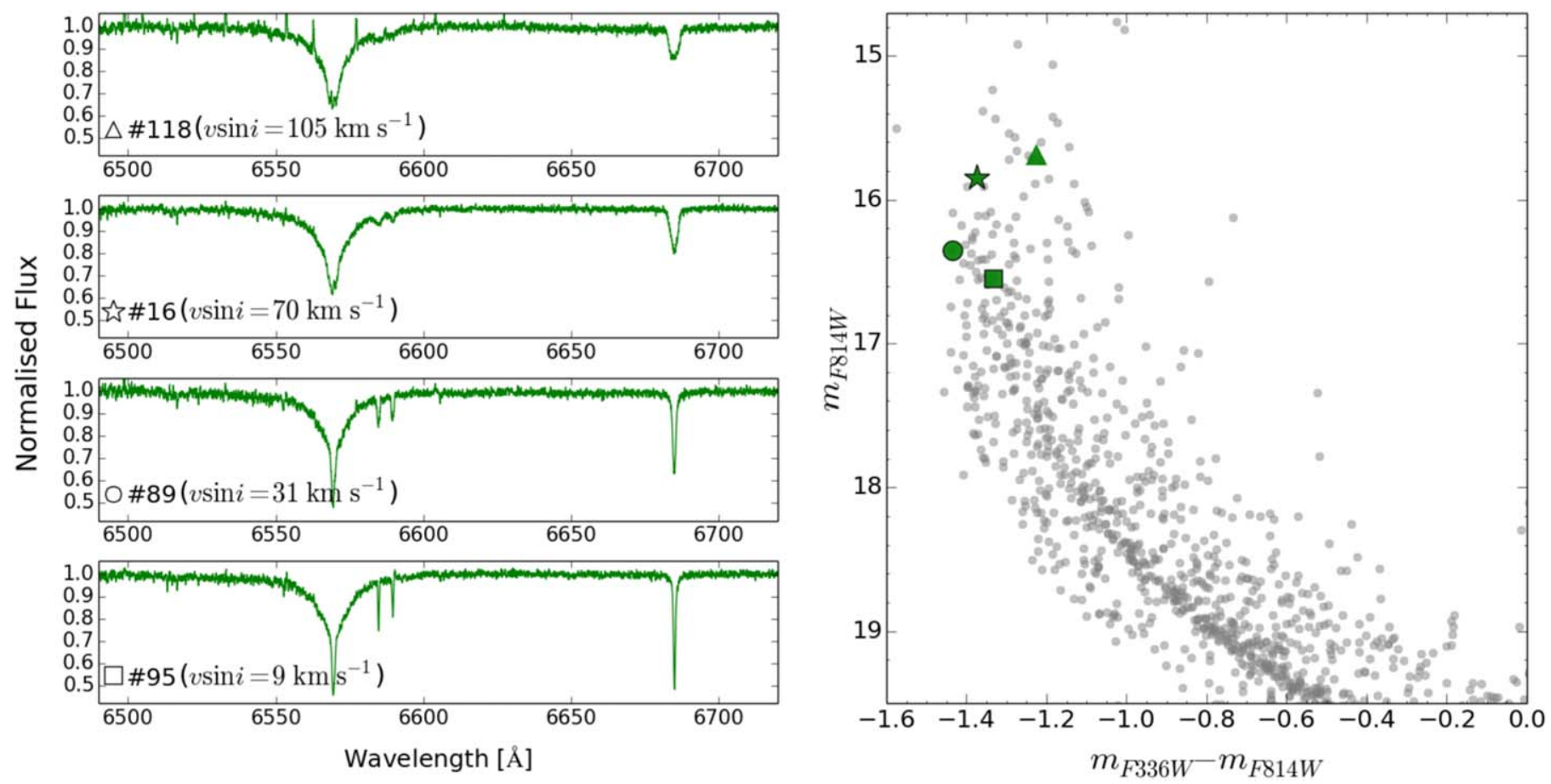

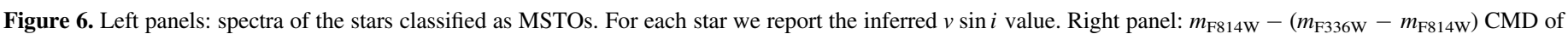

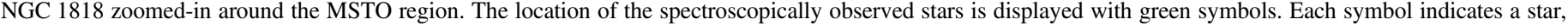
corresponding to the symbols reported in the spectra in the left panels.

with values close to the bMS stars. We suggest caution, however, in the interpretation of the these fainter stars, whose errors in $v \sin i$ are much larger.

In the right panels of Figure 5 we analyze the color of the MS stars as a function of the $v \sin i$ values. For these purposes we have drawn a fiducial line by eye that defines the rMS on the $m_{\mathrm{F} 814 \mathrm{~W}}-\left(m_{\mathrm{F} 336 \mathrm{~W}}-m_{\mathrm{F} 814 \mathrm{~W}}\right) \mathrm{CMD}$ (upper-right panel). The difference in color between each analyzed MS star and the fiducial, $\Delta_{m_{\mathrm{F} 336 \mathrm{~W}}-m_{\mathrm{F} 814 \mathrm{~W}}}$, does not show any significant trend with $v \sin i$ (lower right panel).

\subsection{Extended MSTO}

Figure 6 displays the position on the CMD of the four eMSTO stars, and their spectra. The distribution of the inferred $v \sin i$ values for these stars, compared to the bMS and rMS ones, is shown in Figure 7. At least for the objects studied here, none of the eMSTO stars reach $v \sin i$ values higher than $\sim 150 \mathrm{~km} \mathrm{~s}^{-1}$, which is the limiting value above which we do not find any bMS star. Of course, this does not necessarily mean that none of the analyzed eMSTOs are the counterparts of rMS stars, as below $\sim 150 \mathrm{~km} \mathrm{~s}^{-1}$ we observe both rMSs and bMSs.

\subsection{Be Stars}

Figure 8 displays the spectra of the four stars selected as $\mathrm{Be}$ stars. The top right panel illustrates the position of all the $\mathrm{Be}$ candidates on the $m_{\mathrm{F} 656 \mathrm{~N}}-\left(m_{\mathrm{F} 656 \mathrm{~N}}-m_{\mathrm{F} 814 \mathrm{~W}}\right) \mathrm{CMD}$, e.g., $\mathrm{Be}$ stars are those with an excess in $m_{\mathrm{F} 656 \mathrm{~N}}$ due to the emission in the $\mathrm{H} \alpha$. We have selected the four Be candidates based on their locations on the $m_{\mathrm{F} 656 \mathrm{~N}}-\left(m_{\mathrm{F} 656 \mathrm{~N}}-m_{\mathrm{F} 814 \mathrm{~W}}\right) \mathrm{CMD},{ }^{9}$ hence our spectra in turn confirm that the stars identified as Be on our

\footnotetext{
9 As noted by Dupree et al. (2017), relying on the HST photometry using the narrow filter $m_{\mathrm{F} 656 \mathrm{~N}}$ to identify the Be stars might result in missing some of them, given the high radial velocities of our stars.
}

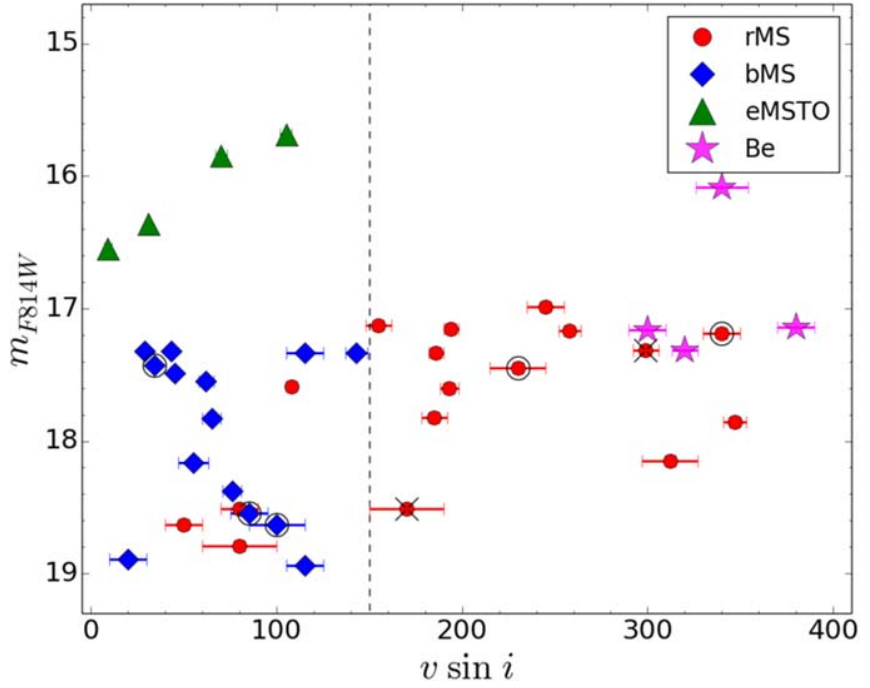

Figure 7. Magnitude $m_{\mathrm{F} 814 \mathrm{~W}}$ as a function of $v \sin i\left[\mathrm{~km} \mathrm{~s}^{-1}\right]$ for rMS (red circles), bMS (blue diamonds), eMSTO (green triangles), and Be stars (magenta stars).

CMD have $\mathrm{H} \alpha$ emission. The bottom right panel of Figure 8 shows that on the $m_{\mathrm{F} 814 \mathrm{~W}}-\left(m_{\mathrm{F} 336 \mathrm{~W}}-m_{\mathrm{F} 814 \mathrm{~W}}\right) \mathrm{CMD}, \mathrm{Be}$ stars are spread over a relatively wide range in color at $m_{\mathrm{F} 814 \mathrm{~W}}$, brighter than $\sim 18.5$. As noted in Milone et al. (2018), nearly all of them are either located on the rMS or populate the reddest part of the eMSTO.

As in Rivinius et al. (2013) Be stars are "very rapidly rotating main sequence B stars, which, through a still unknown, but increasingly constrained process, form an outwardly diffusing gaseous, dust-free Keplerian disk." Hence, the shape of the $\mathrm{H} \alpha$ profile gives us information on the inclination $i$. Star \#84, which is the brightest in $m_{\mathrm{F} 656 \mathrm{~N}}$, is the one that appears 

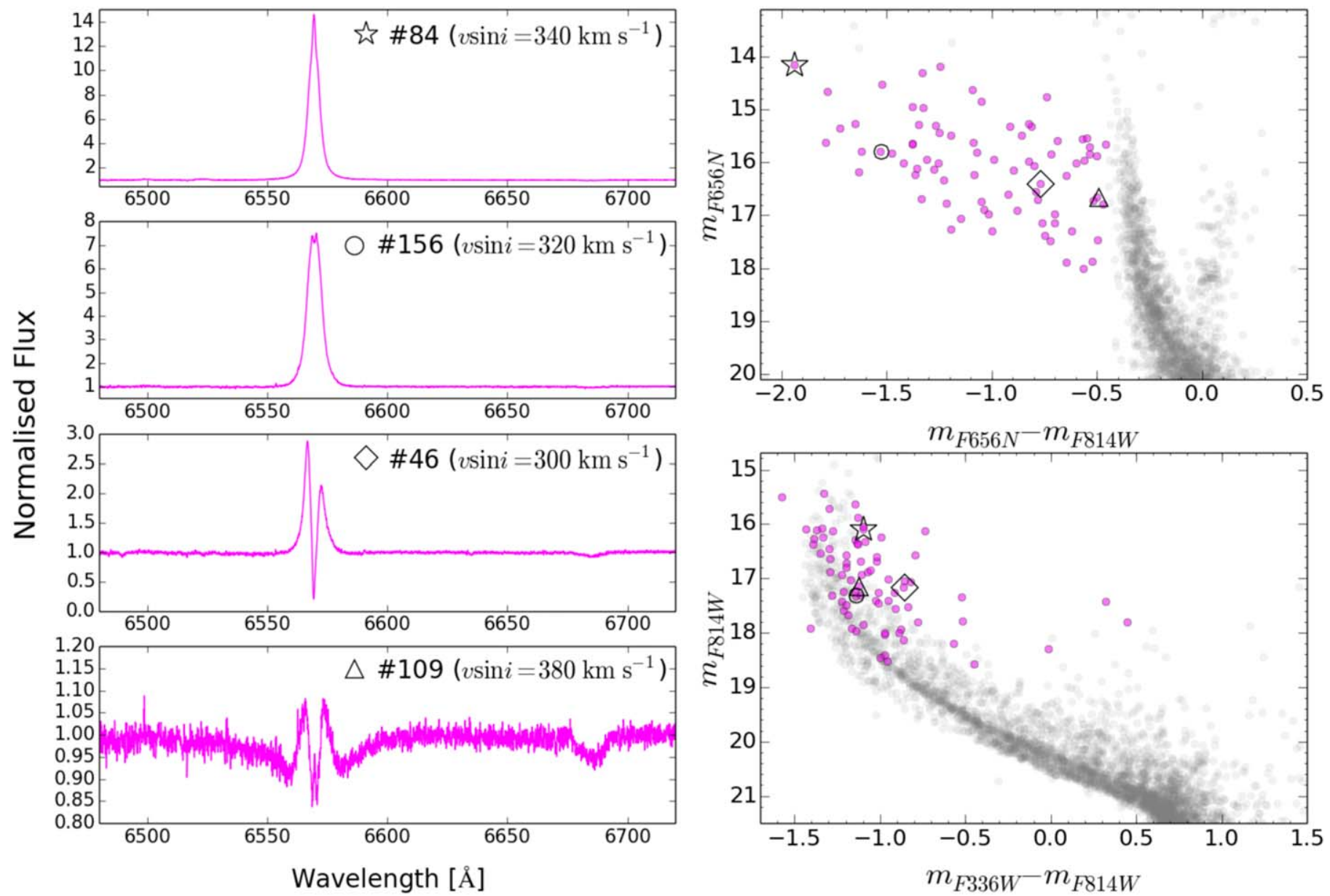

Figure 8. Spectra of the four Be stars in NGC 1818 (left panel), and their locations plotted with different black open symbols on the $m_{\mathrm{F} 656 \mathrm{~N}}-\left(m_{\mathrm{F} 656 \mathrm{~N}}-m_{\mathrm{F} 814 \mathrm{~W}}\right)$ (top right panel) and $m_{\mathrm{F} 814 \mathrm{~W}}-\left(m_{\mathrm{F} 336 \mathrm{~W}}-m_{\mathrm{F} 814 \mathrm{~W}}\right)$ (bottom right panel) CMDs. From top to bottom the spectra represent stars with higher excesses in $m_{\mathrm{F} 656 \mathrm{~N}}$, corresponding to more pronounced emission in $\mathrm{H} \alpha$. In both CMDs all the selected Be stars have been plotted as magenta dots.

closer to a pole-on orientation, as it has a strong narrow emission, and no absorption. Star \#156 also shows a $\mathrm{H} \alpha$ morphology consistent with a near pole-on view, though with a reduced emission. The double-peaked $\mathrm{H} \alpha$ profiles with central absorption of the other Be stars, \#46 and \#109, imply a higher $i$, i.e., we see these objects through their disks (edge-on).

As discussed in Section 3, the $v \sin i$-determination for the Be stars has to rely solely on He I $6678 \AA$. The values inferred are the highest in our sample, all of them $\gtrsim 300 \mathrm{~km} \mathrm{~s}^{-1}$ (see Figure 7). While it is expected that Be stars are fast rotators, the more pole-on views of \#86 and \#156 suggest that they will likely rotate very close to their critical velocities. We also note that star \#46 has an asymmetric He I profile, which either could be a hint of a second light component (the secondary in a physical binary, or a chance alignment) or the signature of inhomogeneities in the disk.

\subsection{Binaries}

We did not attempt any $v \sin i$ determination for stars displaying a double-lined spectrum. These stars could be either physical binaries or alignments of stars. However, it could be interesting to investigate the positions of these stars on the CMD.

Figure 9 shows the location of the double-lined stars on the $m_{\mathrm{F} 814 \mathrm{~W}}-\left(m_{\mathrm{F} 336 \mathrm{~W}}-m_{\mathrm{F} 814 \mathrm{~W}}\right) \mathrm{CMD}$ of NGC 1818 . Two of them, \#40 and \#130, indeed occupy the sequence of binaries that is redder than the rMS. On the other hand, star \#36 is located in the rMS region; as discussed above we expect some contamination between the rMS and the binary regions. The more luminous star, \#131, could be classified as an MSTO.

\section{Summary and Discussion}

We have derived projected rotational velocities $v \sin i$ for rMS and bMS, eMSTO, and Be stars in the young cluster NGC 1818. The presented analysis suggests the following:

1. a clear difference in the mean $v \sin i$ between bMS and rMS stars, with average values of $\langle v \sin i\rangle_{\mathrm{bMS}}=71 \pm$ $10 \mathrm{~km} \mathrm{~s}^{-1}\left(\sigma=37 \mathrm{~km} \mathrm{~s}^{-1}\right)$ and $\langle v \sin i\rangle_{\mathrm{rMS}}=202 \pm$ $23 \mathrm{~km} \mathrm{~s}^{-1}\left(\sigma=91 \mathrm{~km} \mathrm{~s}^{-1}\right)$, respectively;

2. a lower average $v \sin i\left(\langle v \sin i\rangle=54 \pm 25 \mathrm{~km} \mathrm{~s}^{-1}\right)$ for the four stars classified as eMSTO, more consistent with the values measured for the bMS stars; and

3. high $v \sin i \quad\left(\right.$ average $\left.\langle v \sin i\rangle=335 \pm 20 \mathrm{~km} \mathrm{~s}^{-1}\right)$, the highest in our sample, for the analyzed Be stars.

Our results provide the first direct evidence from spectroscopy of different rotation rates for the two MSs observed in a young cluster.

Although $v \sin i$ values are lower limits to the real surface rotational velocity of the stars, they allow us to place some 

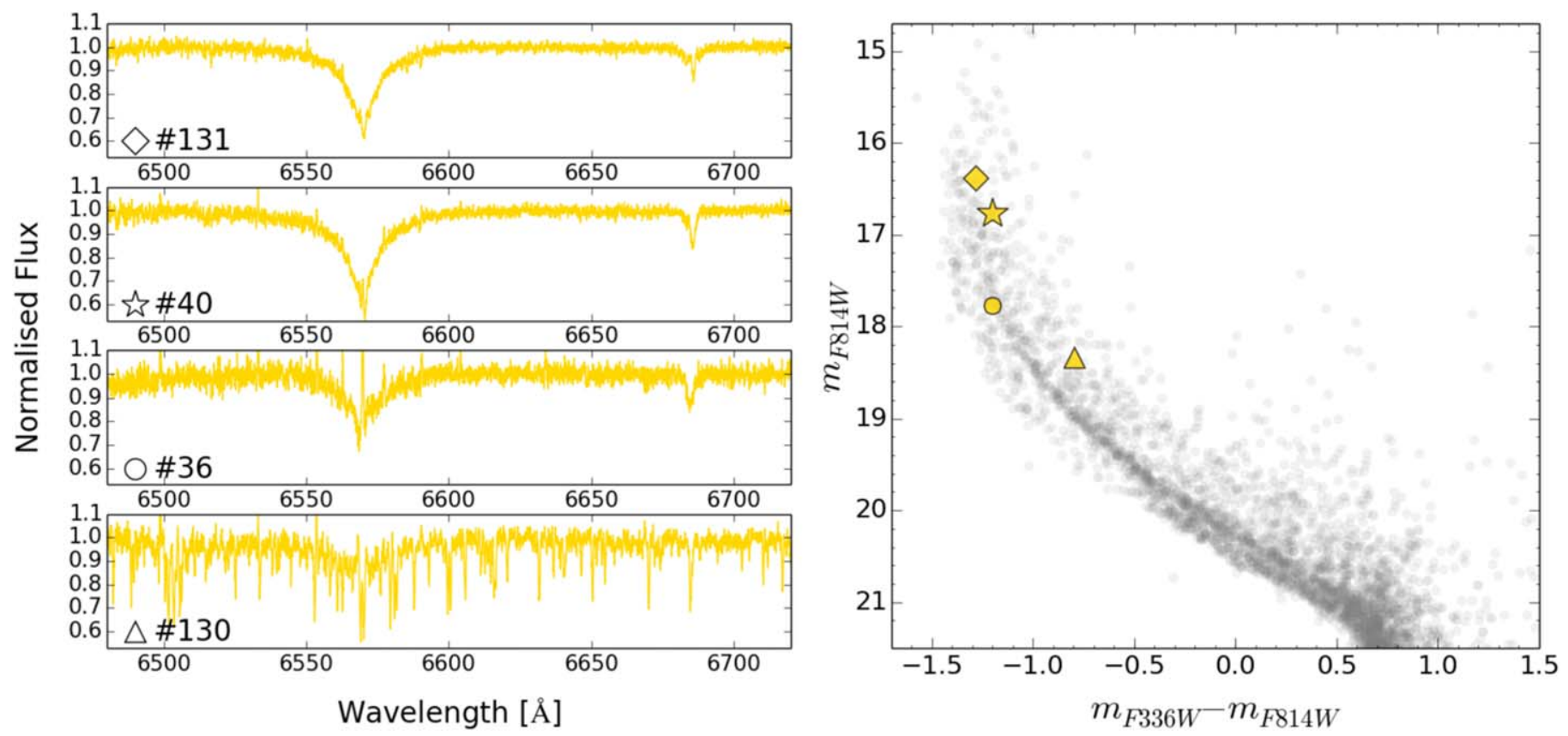

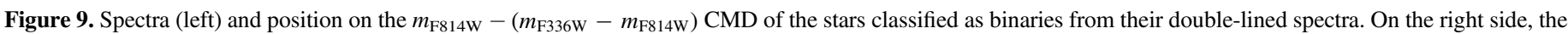
symbols on the CMD correspond to the spectra on the left side.

constraints on the multiple stellar populations observed in young star clusters, specifically in NGC 1818 . The different mean $v \sin i$ derived for bMS and rMS stars confirms the suggestion coming from the analysis of high-precision CMDs from HST that the split MS of young GCs is connected with different rotational regimes. Indeed, Milone et al. (2018) have shown that the CMD of NGC 1818 is best-fitted by isochrones with rotation $\omega=0$ (representative of models with low rotation velocity) to reproduce the bMS, and with $\omega=0.9 \omega_{\text {crit }}$ (representative of high initial rotation) to fit the rMS (see their Figure 10 for the isochrone fit to the CMD).

The Geneva models for $\omega=0.9 \omega_{\text {crit }}$ corresponding to the masses evolving in this cluster $\left(\sim 8 M_{\odot}\right)$ display surface rotation velocities of $330-340 \mathrm{~km} \mathrm{~s}^{-1}$ during the MS lifetime (Georgy et al. 2014), consistent with the maximum $v \sin i$ in our sample, $347 \pm 6$ measured for the rMS star \#141, with the hypothesis that this star has an inclination close to $90^{\circ} .{ }^{10}$ The lower values of $v \sin i$ that we derived for the other stars are consistent with velocities of $\sim 330-340 \mathrm{~km} \mathrm{~s}^{-1}$ and an inclination $<90^{\circ}$.

By simply scaling down the maximum $v \sin i$ observed for our MS stars and assuming that it corresponds to $\omega=0.9 \omega_{\text {crit }}$, the average $v \sin i$ of the bMS stars $\left(71 \mathrm{~km} \mathrm{~s}^{-1}\right)$ could correspond to a value of $\omega \sim 0.2 \omega_{\text {crit }}$. Although this is higher than zero, this is indeed a quite low velocity, still compatible with the location of the bMS, if one looks at the Geneva isochrone location. However, given the average $v \sin i$ values are lower limits to the real stellar rotation, it is likely that the bMS is populated by stars with $\omega>0.2 \omega_{\text {crit }}$.

It has been suggested that the bMS is composed of "braked" stars, and not simply of stars born as slow rotators (D'Antona et al. 2017). In this scenario, the upper MS stars are assumed to

\footnotetext{
${ }^{10}$ Actually, these surface velocities correspond to a ratio $\omega \sim 0.8 \omega_{\text {crit. }}$ Such a difference between the formal value given in the database and the actual value in the models is due to the passage from solid-body rotation assumed in the initial models before the ZAMS to differential rotation on the MS. An equilibrium profile of $\omega$ is built inside the star at the expense of the surface rotation velocity (S. Ekström 2018, private communication).
}

be "nuclearly" younger (since they define a younger isochrone; Milone et al. 2018), because for most of their MS lifetime, they have been subject to the core-envelope mixing of rapidly rotating stars.

Our bMS $v \sin i$ values show a possible increase of rotation toward fainter magnitudes, which, if real, would be consistent with the braking hypothesis, because approximately above the TO of the non-rotating isochrone, we only find almost fully braked stars on the MS; otherwise they would have already evolved. The same reasoning also applies to our four eMSTO stars: none show a $v \sin i$ as high as that of the rMS, possibly suggesting a lack of fast rotators below the highest luminosity turnoff. Although our conclusions are based on a small sample (four stars), a hint of lower velocity for dimmer magnitudes is suggested from our $v \sin i$ of eMSTO stars. This is expected if the eMSTO is interpreted in terms both of braking and of different rotation rates, but note that the post-TO evolution itself implies a braking of the envelope.

Clearly, the analyzed Be stars are the fastest rotators in our sample, with $v \sin i$ being overall higher than those observed along the rMS. In all of them we detect strong $\mathrm{H} \alpha$ emission, clearly indicating a fast-rotating star with a Keplerian decretion disk (Rivinius et al. 2013). The generally higher $v \sin i$ values for the Be stars suggest that these objects are the closest to the breaking speed rotation in the cluster. An increase of the ratio $\omega / \omega_{\text {crit }}$ occurs during the MS lifetime in rotational models, although the equatorial velocity is reduced, and this may be the reason for the formation of the decretion disk.

Qualitatively, our work strongly suggests the deep influence of stellar rotation on the appearance of multiple and/or spread photometric sequences and on shaping the CMDs of young stellar clusters. In more detail, our measurements agree with a rotation for the rMS of about $\omega=0.8 \omega_{\text {crit }}$, which in fact is the rotation rate in the detailed Geneva models formally labeled $\omega=0.9 \omega_{\text {crit }}$ (see footnote 9). A rotation much closer to the critical value might be attributed to the Be stars. We also find a non-zero rotation for bMS stars, which rotate at $\omega \gtrsim 0.2 \omega_{\text {crit }}$, which is much slower 
than the rotation of rMS stars. We also note that the larger spread in the $v \sin i$ values for rMS stars, with respect to the bMSs, might only be partially ascribed to the different inclinations $i$ coupled with high intrinsic rotations, and we cannot exclude some spread in the stellar rotation for these stars.

The presence of split MS in young clusters is one of the most-intriguing and unexpected recent discoveries in the field of stellar populations. This work needs to be seen as the first exploration of some phenomena that could affect not only the cluster formation but also stellar-evolution modeling. Further studies of the rotation velocities in additional clusters, with equivalent or larger samples, will be crucial to fully understand what is clearly a complex phenomenon.

The authors are grateful to the anonymous referee for useful discussions. A.F.M. and G.D.C. acknowledge support by the Australian Research Council through Discovery Early Career Researcher Award DE160100851 and Discovery project DP150103294. A.P.M. has been supported by the European Research Council through the Starting Grant "GALFOR" and the FARE-MIUR project R164RM93XW "SEMPLICE."

Software: ATLAS9 (Kurucz 1993), IRAF (Tody 1986, 1993), DETAIL, SURFACE (Giddings 1981; Butler \& Giddings 1985), SPAS (Hirsch 2009).

\section{ORCID iDs}

A. F. Marino (ib https://orcid.org/0000-0002-1276-5487

A. P. Milone (i) https://orcid.org/0000-0001-7506-930X

G. Da Costa (iD https://orcid.org/0000-0001-7019-649X

A. Dotter (iD https://orcid.org/0000-0002-4442-5700

A. Dupree (iD https://orcid.org/0000-0002-8985-8489

\section{References}

Ahmed, A., \& Sigut, T. A. A. 2017, MNRAS, 471, 3398

Anderson, J., Sarajedini, A., Bedin, L. R., et al. 2008, AJ, 135, 2055

Bastian, N., \& de Mink, S. E. 2009, MNRAS, 398, L11

Bastian, N., Lamers, H. J. G. L. M., de Mink, S. E., et al. 2013, MNRAS, 436, 2398

Bedin, L. R., Cassisi, S., Castelli, F., et al. 2005, MNRAS, 357, 1038

Butler, K., \& Giddings, J. R. 1985, Newsletter of Analysis of Astronomical Spectra, No. 9 (Univ. London)

Conroy, C., \& Spergel, D. N. 2011, ApJ, 726, 36
Correnti, M., Goudfrooij, P., Bellini, A., Kalirai, J. S., \& Puzia, T. H. 2017, MNRAS, 467, 3628

D’Antona, F., Di Criscienzo, M., Decressin, T., et al. 2015, MNRAS, 453, 2637

D’Antona, F., Milone, A. P., Tailo, M., et al. 2017, NatAs, 1, 0186

D'Antona, F., Vesperini, E., D'Ercole, A., et al. 2016, MNRAS, 458, 2122

Decressin, T., Meynet, G., Charbonnel, C., Prantzos, N., \& Ekström, S. 2007, A\&A, 464, 1029

Dupree, A. K., Dotter, A., Johnson, C. I., et al. 2017, ApJL, 846, L1

Firnstein, M., \& Przybilla, N. 2012, A\&A, 543, A80

Frémat, Y., Zorec, J., Hubert, A.-M., \& Floquet, M. 2005, A\&A, 440, 305

Gaia Collaboration, Brown, A. G. A., Vallenari, A., et al. 2016, A\&A, 595, A2

Georgy, C., Granada, A., Ekström, S., et al. 2014, A\&A, 566, A21

Giddings, J. R. 1981, PhD thesis, Univ. London

Girardi, L., Rubele, S., \& Kerber, L. 2009, MNRAS, 394, L74

Glatt, K., Grebel, E. K., Sabbi, E., et al. 2008, AJ, 136, 1703

Goudfrooij, P., Girardi, L., Kozhurina-Platais, V., et al. 2014, ApJ, 797, 35

Hirsch, H. A. 2009, PhD thesis, Univ. Erlangen-Nürnberg

Keller, S. C., Mackey, A. D., \& Da Costa, G. S. 2011, ApJ, 731, 22

Korn, A. J., Keller, S. C., Kaufer, A., et al. 2002, A\&A, 385, 143

Kurucz, R. L. 1993, CD-ROM No. 13 (Cambridge, Mass.: SAO)

Mackey, A. D., \& Broby Nielsen, P. 2007, MNRAS, 379, 151

Marino, A. F., Milone, A. P., Casagrande, L., et al. 2016, MNRAS, 459, 610

Marino, A. F., Villanova, S., Piotto, G., et al. 2008, A\&A, 490, 625

McLaughlin, D. E., \& van der Marel, R. P. 2005, ApJS, 161, 304

Milone, A. P. 2015, MNRAS, 446, 1672

Milone, A. P., Bedin, L. R., Piotto, G., et al. 2015a, MNRAS, 450, 3750

Milone, A. P., Bedin, L. R., Piotto, G., \& Anderson, J. 2009, A\&A, 497, 755

Milone, A. P., Marino, A. F., D'Antona, F., et al. 2016, MNRAS, 458, 4368

Milone, A. P., Marino, A. F., D’Antona, F., et al. 2017a, MNRAS, 465, 4363

Milone, A. P., Marino, A. F., Di Criscienzo, M., et al. 2018, MNRAS, 477, 2640

Milone, A. P., Marino, A. F., Piotto, G., et al. 2012, ApJ, 745, 27

Milone, A. P., Marino, A. F., Piotto, G., et al. 2015b, ApJ, 808, 51

Milone, A. P., Piotto, G., Renzini, A., et al. 2017b, MNRAS, 464, 3636

Nelder, J. A., \& Mead, R. 1965, CompJ, 7, 308

Nieva, M. F., \& Przybilla, N. 2007, A\&A, 467, 295

Pasquini, L., Avila, G., Blecha, A., et al. 2002, Msngr, 110, 1

Przybilla, N. 2005, A\&A, 443, 293

Przybilla, N., \& Butler, K. 2004, ApJ, 609, 1181

Przybilla, N., Nieva, M.-F., \& Butler, K. 2011, JPhCS, 328, 012015

Renzini, A., D’Antona, F., Cassisi, S., et al. 2015, MNRAS, 454, 4197

Rivinius, T., Carciofi, A. C., \& Martayan, C. 2013, A\&ARv, 21, 69

Smith, K. C. 1996, Ap\&SS, 237, 77

Tody, D. 1986, Proc. SPIE, 627, 733

Tody, D. 1993, in ASP Conf. Ser. 52, Astronomical Data Analysis Software and Systems II, ed. R. J. Hanisch, R. J. V. Brissenden, \& J. Barnes (San Francisco, CA: ASP), 173

Townsend, R. H. D., Owocki, S. P., \& Howarth, I. D. 2004, MNRAS, 350, 189

Wade, R. A., \& Rucinski, S. M. 1985, A\&AS, 60, 471

Wilson, C. P. 1975 , AJ, 80, 175 